Nataša GAVRILOVIĆ*

Università di Belgrado

\title{
LA TRASPOSIZIONE LETTERARIA DELLA REALTÀ STORICA NELLE LETTERE DI GIAMBATTISTA MARINO
}

Parole chiave: Giambattista Marino, lettere, documento storico vs retorica dell'epistola.

Nel 1620, in una delle sue lettere, il Marino scrive all'amico Barbazza dell'idea di raccogliere «un volume di lettere che non saranno forse ordinarie», diviso «in lettere gravi, familiari, amorose e burlesche». ${ }^{1}$ Il poeta però non vedrà il progetto andare in porto, saranno gli altri a raccogliere le lettere, pubblicandole due anni dopo la sua morte.

Comunque sia, l'intento di fare un insieme delle proprie, tra l'altro assai numerose lettere e di pubblicarle esisteva nella mente del poeta, e non è un dato irrilevante, soprattutto per il Marino «sensibilissimo all'atto irrevocabile della stampa». ${ }^{2}$ Subito dopo, il Marino continua: «e in esse non mi scorderò d'honorarvi gli amici, ma spetialmente voi, che siete tra cari il carissimo». ${ }^{3} \mathrm{Si}$ intravedono così alcuni dei punti principali dell'idea dell'epistolario mariniano - diverse tonalità delle lettere (appunto perché individuate come gravi, familiari, amorose, burlesche, ecc) e la presenza soprattutto degli amici, come complici, e nel contempo come conferma della sua vita gloriosa, dai quali naturalmente dipenderà il tono stesso della lettera. La creazione dell'epistolario diventa ancor più interessante

*natascia1327@gmail.com

${ }^{1}$ G. Marino, Lettere, a cura di M. Guglielminetti, Torino, Einaudi, 1966, p. 269.

Cfr. A. Battistini, Lo specchio di Dedalo, Bologna, il Mulino, 1990, pp. 27-29.

${ }^{2}$ E. Russo, Una nuova redazione del «Ragguaglio a Carlo Emanuele» del Marino, «Filologia italiana», 7, 2010, p. 109.

${ }^{3}$ Marino, Lettere, cit., p. 269. 
se riteniamo veritiero il dato che prima di morire il Marino abbia bruciato alcune lettere, ${ }^{4}$ poiché anche un tale gesto potrebbe essere interpretato come una specie di selezione tra il proprio corpus epistolare, selezione che è pur sempre un riflesso del chiaro intento del poeta.

Tenendo conto di ciò, ma anche della stessa forma epistolare - già di per sé assai interessante in quanto forma aperta e come genere che, accanto all'autobiografia, forse più si avvicina al confine tra la verosimiglianza e la verità - occorre procedere con cautela nella lettura dell'epistolario mariniano. Anche se potrebbe essere di grande utilità per ricostruire la biografia e la poetica dell'autore, va tenuto presente il fatto che la realtà storica traspostavi sia una realtà filtrata, specchio delle idee e delle aspirazioni dell'autore, il quale fa sì che, al posto della sua persona "vera" e "reale", davanti a noi si delinei anzitutto il personaggio che lo stesso Marino vuole mostrare, un ritratto verosimile all'idea e, si potrebbe dire, all'ideale della propria personalità. ${ }^{5}$ Proprio nella scelta e quindi nell'omissione di certe lettere, al fine della coerenza dell'unità dell'epistolario, ma anche dell'unità della propria immagine che in esso viene creata, si rispecchia la perizia letteraria del Marino, del Marino prosatore innanzitutto (ma non solo, come vedremo), e la funzione della forma epistolare, attraverso la quale questa perizia si manifesta.

La natura privata e quella pubblica del testo, due discorsi ben diversi, stabiliscono un dialogo assai complesso, comportando dei cambiamenti nel passaggio dall'uno all'altro. Inoltre, dietro il gioco dei due discorsi si nasconde quello ancor più intricato: il rapporto realtà-finzione. Sarà la forma ${ }^{6}$ a dare all'evento storico la plusvalenza letteraria che lo rende ancor più vero, sottoponendo la già menzionata verità alla verosimiglianza, il che, nelle lettere, si riverbera prima di tutto attraverso l'adeguamento del contenuto alla forma stessa e ai destinatari. Per vedere come tutto ciò funziona, ci siamo limitati ad un solo e il più drammatico evento nella sua cruda verità biografica - il famoso attentato organizzato e messo in atto

${ }^{4}$ Cfr. M. Zogović, Barok: knjževna teorija i praksa, Beograd, Narodna knjiga - Alfa, 2007, p. 120.

${ }^{5}$ Cfr. l'osservazione di Sandro Briosi: «L'autobiografo, diceva infatti Rousseau, "si mostra come vuole esser visto". Certo, ma non si tratta di ipocrisia, bensì di immaginazione. L'opposizione non si dà tra il "come vuol esser visto" ed un inesistente "come è realmente"; si pone tra il "come vuol esser visto" e il "come è visto" dallo sguardo che radica e insieme aliena nelle storia, degli Altri» (S. BRIOsI, Autobiografia e finzione, «Quaderni di retorica e poetica: rivista semestrale di retorica e poetica del Circolo filologico linguistico padovano», I, 1985, pp. 9-16).

${ }^{6}$ Anzi, le forme, poiché lo stesso evento verrà esposto anche nelle ottave del poema epico mariniano, come in seguito si vedrà. 
da Gasparo Murtola, avvenuto a Torino nel 1609 - e alle lettere a diversi destinatari che vi sono dedicate (lettere nn. 47, 48, 49, 50, 51 nell'edizione consultata).

«Una lettera in forma di manifesto intorno a questa materia [l'attentato, N.d.A], scritta da me non per altro che per mia discolpa» ${ }^{7}$ - è così che viene presentata nella missiva a Fortunato Sanvitale la lettera più lunga, una delle più famose, e forse la più interessante dal punto di vista linguisticostilistico e retorico - quella del 15 febbraio 1609, diretta da Torino a Carlo Emanuele I di Savoia. La lettera, infatti, sarà davvero una specie di manifesto dell'evento, completamente intriso di osservazioni soggettive e personali, retoricamente assai complesso, come ogni manifesto, ma capace, proprio in virtù di tale retoricità ed elaborazione stilistica, ad innalzare la verità storica all'altezza della verità assoluta. Lo stesso Marino sottolinea e spiega che si tratta della «verità del fatto». ${ }^{8}$ Vi si espongono con dovizia di dettagli le circostanze e gli eventi che precedono l'attentato e quello che avviene in seguito, ma anche le possibili cause così come gli argomenti che servono da difesa del proprio onore, per concludere, tuttavia, chiedendo il perdono per Murtola, e riuscendo persino ad ottenerlo. Elaborato così, l'evento si trasforma davvero «da un fatto di cronaca nera a ritratto morale interiore». ${ }^{9}$ E non solo. Il registro prescelto e l'elaborazione linguistico-stilistica saranno, a nostro avviso, decisivi per la sua rappresentazione. Infatti, la lettera è del tutto permeata di vari virtuosismi linguistici e stilistici, di varie figure il cui iperonimo si trova nella ingegnosità: una vasta presenza di figure etimologiche, paronomasie, paradossi, ossimori, parallelismi sintattici, con la sintassi assai complessa, periodi lunghi e tante proposizioni incidentali.

Inoltre, la figura retorica abbondantemente usata è non a caso il topos della falsa modestia. Ne offriamo qualche esempio:

${ }^{7}$ Marino, Lettere, cit., p. 96.

${ }^{8}$ Citiamo l'intero passo della lettera: «Onde io, non già per accrescere con la repulsa delle sue menzogne la gravezza del delitto, poiché, avendo compassione alla sua pazzia, oltre l'avergli perdonato, mi sono con molti mezzi adoperato per far che ottenga la grazia, ma solamente per purgar la mia riputazione di qualsivoglia macchia, ed accioché $\mathrm{V}$. A. non bene informata del vero non mi accusi d'immodestia e non mi dia titolo d'arrogante, sotto pretesto ch'io abbia altrui insolentemente provocato con libelli oltraggiosi; ho stimato necessario con questa lettera dichiarare apertamente la verità del fatto, divisando con ogni confidenza e simplicità a lei, non come a giudice ma come a prencipe, tutto quel progresso di cose ch'al fisco, per dubbio di non aver a nuocer al reo, ho voluto tacere» (MARINO, Lettere, cit., p. 77).

${ }^{9}$ Battistini, Lo specchio di Dedalo, cit., p. 28. 
Dico adunque ch'io delle risse e delle dissensioni non mi sono dilettato giammai, anzi ingegnandomi con ogni sforzo, secondo la bassezza dello stato mio, non di spiacere ma di servire altrui, fui sempre, più che degli studi stessi, della pace studioso. Né questo voglio io attribuire a virtù morale, abito della elezione, ma a propria condizione della mia natura, amica della tranquillità;

[...] onde mi trasse di bocca parole sconce ed assai diverse da quella modestia della qual feci sempre professione;

E se non sapessi ch'io son suggetto di niuna levatura, direi ch'egli, con l'essempio di colui il quale con l'incendio del tempio di Efeso pensò d'acquistarsi un grido eterno, tentasse d'eternarsi con la mia distruzione;

E quantunque io non sia in tale stato, che né per merito né per grandezza possa o debba essere invidiato, tale è nondimeno la meschinità degli infelici, che suole alle volte insidiare anche le picciole fortune di chi almeno sopra loro s'avanza. ${ }^{10}$

Oltre al topos retorico della falsa modestia, vi sono altri passi più vistosamente permeati di riferimenti biografici, modellati magari per il più vasto pubblico che avrà l'occasione di leggere/sentire la lettera, dato che lo stesso poeta ne dichiara l'intenzione. ${ }^{11} \mathrm{Ne}$ citiamo alcuni:

Io seguitando pur la solita libertà del mio genio, gli risposi quello stesso che sempre dissi di costui $[\ldots]$;

Mosso intanto il signor conte di Passano dal zelo della quiete commune, venne instantemente a sforzarmi, non che a pregarmi, ch'io con esso lui mi rappattumassi; ed a me che il mostrar ritrosia nelle cose oneste riputai sempre costume zotico, piacque per più rispetti alle sue essortazioni consentire;

[...] e dico ch'a queste ultime scosse non fu possibile star saldo, ma stuzzicato da tante impertinenze mi fu forza cedere all'ira, dar bando al rispetto e deporre pur un tratto parte della mia solita flemma. ${ }^{12}$

Si potrebbe concludere, insieme a Battistini, che il codice retorico prevale sul messaggio ${ }^{13}$ - può darsi addirittura che in esso stia il vero messaggio della lettera. Ciò diventa ancor più lampante se consideriamo

${ }^{10}$ MARINO, Lettere, cit., pp. 77-95.

${ }^{11}$ «E voglio che questa mia protesta quando mi parrà tempo si publichi, perché intendo che in ogni caso alla giornata mi giovi e pretendo che contro la perversità de' miei avversari debba esser propugnacolo e scudo», MARINO, Lettere, cit., p. 85, corsivo N.G.

${ }^{12}$ Marino, Lettere, cit., pp. 79-86, corsivo N.G.

${ }^{13}$ Battistini, Lo specchio di Dedalo, cit., p. 29. 
le altre lettere dedicate allo stesso evento. ${ }^{14}$ Il loro tono più "familiare" e la minor elaborazione linguistico-stilistica creano una netta differenza tra le lettere fin da subito concepite come pubbliche e altre, annoverate più tardi nell'epistolario "ufficiale", oppure rigettate. ${ }^{15}$ Tuttavia, anch'esse fanno parte di un unico mosaico della figura pubblica del Marino, poiché in tutte le rappresentazioni dell'evento, a prescindere dai destinatari, c'è un solo punto di vista - il punto di vista di un poeta, anzi del Poeta. Il fulcro della lettera-manifesto al Duca, che è anzitutto un'autodifesa, sta nella difesa delle proprie opere riguardo alle loro presunte scurilità e lascivie. ${ }^{16}$ In altre lettere, l'attentato assume una posizione quasi marginale, e ha valore soltanto nella misura in cui influisce sulla creazione del poeta napoletano; in quelle dirette al San Vitali e al Barbazza, persino con le stesse identiche frasi, il traumatico evento sarà citato come causa principale della sua poca produttività, della quale si scusa con gli amici, mentre in un'altra missiva, sempre al San Vitali, dopo la breve descrizione dell'attentato, il Marino conclude tornando alle proprie poesie: «Or, poiché la cosa è fatta, manderò quanto prima a V. S. il rimanente delle altre Fischiate le quali voglio che si veggano». ${ }^{17}$

${ }^{14}$ Occorre dire che gli altri destinatari sono gli amici del Marino - F. San Vitali, letterato e pittore, membro dell'Accademia degli Innominati, A. Barbazza, uno dei protagonisti della vita letteraria della sua città natale - Bologna, membro di due accademie di Bologna - dell'Accademia degli Indomiti e dell'Accademia della Notte, e di una a Venezia - Accademia degli Incogniti, e C. Achillini, giurista, scrittore e poeta, definito marinista.

${ }^{15}$ Cfr. L. MATt, Modelli per l'epistolografia italiana secentesca, «Studi linguistici italiani», XLII, 2016, pp. 241-267. Per darne un esempio, citiamo soltanto la prima frase delle lettere rispettivamente a Sanvitale e al duca di Savoia: «Il Murtola, ancorché si vedesse da me molto strappazzato e beffato con tante Fischiate e si accorgesse d'esser divenuto favola ed obbrobrio non solo della corte, ma di tutta la città, il tutto nondimeno dissimulava; e se bene in apparenza si vedeva turbato, dimostrava però una flemmatica sofferenza»; «Egli mi è pur ora pervenuto a notizia sì come il Murtola, dopo l'aver commesso l'assassinio che si sa nella persona mia e dopo l'essere stato dalla divina giustizia e dal proprio peccato condotto in prigione, per onestar la sua causa e colorir la sua sceleragine con iscuse almento apparenti, ha divulgata una voce, in cui si duole d'essere stato da me offeso nell'onore; e che l'avere io procurato di svergognarlo con rime satiriche, toccando l'onestà delle sue attenenti, è stata la principal cagione che l'ha stimolato a ciò fare».

${ }^{16}$ «Non niego io che per accomodarmi all'umore del secolo, per lusingare l'appetito del mondo e prender lo stile morbido, vezzoso ed atrattivo, non mi sia alquanto dilettato delle amorose tenerezze e che non si possa dalle mie cose raccogliere alcun cenno di metafora, la qual con misteriosa allegoria alluda a qualche lascivo sentimento, appena però penetrabile dagl'intelletti svegliati ed arguti. Niego bene ch'io abbia giammai in esse trattato di cose sozze [...]», MARINO, Lettere, cit., p. 84.

${ }^{17}$ Ivi, p. 76. 
Da questo punto di vista, la missiva forse più interessante sembra la risposta all'Achillini, sempre legata allo stesso evento, in cui il Marino, dopo aver ringraziato l'amico dell'affettuosa lettera, si dedica alla sua analisi stilistico-linguistica e retorica, lodandola come «parto d'arguto e vivace intelletto», piena "di concetti e di vivezze», ${ }^{18}$ ingegnosa. Così, quasi esponendo la propria "poetica epistolare", il Marino palesa la cruciale importanza attribuita al come, al di là e al di sopra del cosa, e in questa direzione continua anche la seguente risposta da parte dell'Achillini. In effetti, dopo ben quattro letture della lettera-manifesto insieme agli amici, l'amico afferma che «non si può leggere senza odiarne il Murtola, amarne V. S. ed ammirare il suo nobilissimo ingegno». ${ }^{19}$ Descritta così, la lettera al Duca sembra piuttosto una vera e propria opera di fantasia e perizia letteraria che, secondo l'Achillini, rappresenta «sì preziosi parti dell'ingegno [...] che forsi passano quante cose fece giamai [il Marino, N.d.A]», trasformando il rapporto Marino-Murtola nel rapporto protagonista-antagonista. $^{20}$

In questa maniera, dalla finzione realistica della forma epistolare ci si avvicina alla finzione vera e propria, e vi si arriva con il capolavoro mariniano, in cui appunto l'evento verrà trasposto. Si tratta del IX canto dell'Adone e dell'incontro con Fileno, l'alter ego del poeta, presso la fontana d'Apollo. Nelle ottave in questione (78-85), naturalmente, l'argomento si adatta alla forma ed al contenuto del poema epico e quindi viene esposto in una maniera molto più concisa ed essenziale rispetto alle lettere. Tuttavia, nella descrizione del percorso, diremmo esemplare, del Poeta, parlando dell'attentato, il Marino non esiterà a usare persino le stesse identiche parole della lettera al Duca, a volte anche intere frasi. ${ }^{21}$ Che un dato autobiografico venga inserito in un'opera letteraria non è certo peculiarità soltanto delle opere mariniane; comunque, quello che, a nostro avviso, risulta curioso è il modo in cui verranno trattate sia le lettere che le ottave menzionate nelle assai numerose biografie ossia Vite del cavalier Marino, con cui inizia quel

${ }^{18}$ Ivi, p. 99.

${ }^{19}$ G. MARIno, Epistolario seguito da lettere di altri scrittori del Seicento, a cura di A. Borzelli e F. Nicolini, vol. II, Bari, Laterza, 1912, p. 123.

${ }^{20}$ Ibid.

${ }^{21}$ Come, ad esempio, fellon per designare il Murtola, fiele e veleno, per indicare l'odio dell'avversario, oppure l'intero verso (ultimo dell'ottava 83) «si volse immortalar con la mia morte», che è identico alla frase della lettera al Duca: «[...] crederei che il Murtola, accorgendosi di non poter vivere nella memoria del mondo, volesse immortalarsi con la mia morte $[\ldots] »$, con la figura etimologica, tanto cara al Marino. 
«processo di mistificazione della sua esistenza», ${ }^{22}$ rafforzato poi, oppure nel contempo, dall'epistolario.

La prima biografia, quella del Baiacca (1625), anche se precede di due anni l'epistolario, abbraccia completamente la versione mariniana del conflitto col Murtola. ${ }^{23}$ Nell'arco di pochi anni escono altre biografie, come quella di Francesco Chiaro (1632), oppure quelle di Francesco Loredano e di Francesco Ferrari, entrambe del 1633. Seguendo l'ordine cronologico, partiamo dalla Vita del cavalier Marino di F. Chiaro. ${ }^{24}$ In essa viene accettata non solo la versione mariniana dell'evento, ma anche il modo in cui il poeta l'aveva rappresentato: vi si trovano intere frasi prese dalle lettere del Marino e ugualmente dalle risposte dei suoi amici. ${ }^{25}$ Ancor più notevole, però, è la presenza delle menzionate ottave autobiografiche che qua vengono prese ed esplicitamente citate come altrettanto valida fonte di informazioni. Lo stesso modo viene accolto anche dal Loredano, ${ }^{26}$ che nella sua biografia include i versi del Marino, ma parafrasandoli: «Fù gratia del Cielo che non permesse un tradimento così esecrabile. L'innocenza è scudo sicuro contro i colpi della perfidia. O'pure fù virtù di quell'alloro, che meritava la sua virtù, che non permesse le ferite di quel fulmine». ${ }^{27}$ Inoltre, insieme alla versione mariniana dell'attentato, il Loredano adotta anche i passi autobiografici da noi individuati come quelli più retorici: «[...] non potendo sofferire quell'arroganza, che gli partoriva la gara virtuosa, $s i$ lasciò cadere dalla bocca alcune parole sconcie, e lontane da'sensi della

${ }^{22}$ M. Guglielminetti, Tecnica e invenzione nell'opera di Giambattista Marino, Messina-Firenze, D’Anna, 1964, p. 51.

${ }^{23}$ Ivi, p. 52.

${ }^{24}$ F. Chiaro, Vita del cavalier Marino descritta dal Signor Francesco Chiaro canonico napolitano suo nipote (https://books.google.rs/books?id=f-JoAAAAcAAJ\&printsec=fron tcover\&hl=it\&source $=$ gbs_ge_summary_r\&cad $=0 \# \mathrm{v}=$ onepage $\& \mathrm{q} \& \mathrm{f}=\mathrm{false})$

${ }^{25}$ Come quella dell'Achillini, ad esempio, cfr. «Trà poeti si guerreggia con le bacche di lauro pianta di Febo, \& non con le ghiande di piombo frutti di Saturno; ma forse il Murtola il cui lauro era sterile, \& infecondo non haveva bacche da gettar via, ricorse per aiuto, al più maligno pianeta, che si raggira per lo Cielo» (Ivi, p. 27). La quasi identica frase la troviamo nella lettera dell'Achillini: "Vuolsi tra poeti guerreggiarsi con le bache del lauro, pianta del Sole; non con le ghiande di piombo, frutti di Saturno. Ma forsi l'aversario, il cui lauro è sterile e infecondo, non avea bacche da gettare; onde ricorse per aiuto al più maligno pianeta che s'aggiri per lo cielo» (MARINo, Epistolario (...), cit., pp. 123-24).

${ }^{26}$ F. LoREDANo, Vita del cavalier Marino di Gio. Francesco Loredano nobile veneto, https://books.google.rs/books?id=GE11AAAAcAAJ\&printsec=frontcover\&hl=it\&source $=\mathrm{gbs}$ ge_summary_r\&cad $=0 \# \mathrm{v}=$ onepage $\& \mathrm{q} \& \mathrm{f}=$ false

${ }^{27}$ Ivi, p. 13. Cfr. i seguenti versi dell'Adone (IX, 85): «Ma fusse pur del ciel grazia seconda/ ch'innocenza e bontà sovente aita/o pur virtù di quella sacra fronda/ che da folgore mai non è ferita». 
sua modestia». ${ }^{28}$ La Vita del Ferrari ${ }^{29}$ rimane, a quanto pare, priva di tanta fedeltà linguistico-stilistica e retorica, pur accettando la versione mariniana dell'evento e quindi mantenendo anche quella vena mistificatoria (sono pur sempre la divina provvidenza e l'Apollo ad avergli salvato la vita). Inoltre, ciò che rende ancor più interessante queste rappresentazioni è il fatto che due secoli dopo le prime biografie pubblicate, anche il Vallauri, filologo ottocentesco, scriva il racconto Il cavalier Marino in Piemonte ${ }^{30}$ seguendo l'esempio dei suoi predecessori, e non solo attinge esclusivamente alle lettere come all'unica fonte d'informazioni, bensì le cita in una tale misura che sembrano piuttosto trasposte che parafrasate.

Una volta narrativizzata e retoricizzata dal Marino, la rappresentazione dell'attentato rimane dunque quasi identica nei testi degli altri. ${ }^{31}$

Il rapporto finzione/realtà diventa quindi ancor più complesso in quanto arricchito da un altro elemento: finzione/storiografia/realtà. Il confine tra questi tre elementi, nel caso del Marino, è assai fragile, a volte quasi invisibile. Ciò risulta del tutto coerente ai propositi, impliciti, del poeta, poiché a unire le diverse forme tra cui il medesimo evento si muove è sempre il modo della sua rappresentazione. Se è il modo il punto fermo, ne discende che i confini tra varie forme (lettera/poema epico mitologico/biografia) e tra vari concetti (storia/finzione/realtà) vengono annullati dalla letterarietà dell'evento e quindi della vita stessa del Marino che vi è rappresentata. Perciò, tornando al nostro punto di partenza, non stupisce la difficoltà di collocare l'epistolario mariniano in uno schema preciso dei «libri di lettere» del Cinquecento oppure dei formulari del Segretario secenteschi. ${ }^{32}$

${ }^{28}$ Ivi, pp. 11-12. Cfr: «onde mi trasse di bocca parole sconce ed assai diverse da quella modestia della qual feci sempre professione» (MARINO, Lettere, cit., p. 78).

${ }^{29}$ F. FERRARI, Vita del cavalier Gio. Battista Marino descritta dal cavaliere Francesco Ferrari, https://books.google.rs/books?id=O2Jd5Urhll4C\&printsec $=$ frontcover\&dq $=$ vita $+\mathrm{d}$ el + cavalier + marino + ferrari\&hl $=i t \& s a=X \& v e d=0$ ahUKEwjBpOu4zsPgAhVoqIsKHbuECR EQ6AEIKDAA\#v=onepage $\& \mathrm{q}=\mathrm{vita} \% 20 \mathrm{del} \% 20$ cavalier $\% 20$ marino $\% 20$ ferrari\&f $=$ false

${ }^{30} \mathrm{~T}$. VAllaURI, Il cavalier Marino in Piemonte: episodio della storia subalpina del secolo XVII, Torino, Stamperia reale, 1847.

${ }^{31}$ Bisogna dire che anche il Boccalini nei suoi Ragguagli di Parnaso (1613) menziona questo episodio adottando la versione e lo stile mariniani.

${ }^{32}$ Proprio parlando della tradizione epistolare in volgare, sarebbe il caso di darne una sintesi, per poter tentare di collocarvi anche le lettere mariniane e avvistarne la particolarità. Il curiosissimo processo di "sviluppo" ed "evoluzione” della forma epistolare nella tradizione letteraria italiana parte dai libri di lettere di Pietro Aretino (il primo libro esce nel 1538), che dominano lungo tutto il Cinquecento, sia in forma di libri di lettere d'autore oppure come raccolta di lettere di uomini eccellenti ed illustri d'epoca (una delle più famose raccolte del genere è quella di Paolo Manuzio, del 1542). All'epoca essi avevano 
Nell'impostazione del Quondam, il quale delimita il campo dei «libri di lettere» con due anni significativi - quello della pubblicazione del primo libro di lettere del divino Aretino (1538) e quello che chiude la "stagione" di questo genere, ovvero l'anno dell'edizione delle lettere dell'altrettanto divino Marino (1627), esse in realtà si trovano al bivio, come «il punto di cerniera tra due esperienze profondamente differenziate»». ${ }^{33}$ Dalla prospettiva delle lettere prese in esame, si potrebbe comunque dire che oltrepassano qualsiasi divisione, visto che tutti gli elementi comuni sia con i libri di lettere che con i formulari, sono anch' essi diventati una peculiarità tutta mariniana, sempre sotto il segno della letterarietà. Il timbro personale, autobiografico, che l'autore vi lascia e il legame con la contemporaneità sono propri sì delle lettere cinquecentesche, però qui in funzione soltanto dell'immagine del Poeta par excellence, e si tratta comunque di una contemporaneità filtrata, ${ }^{34}$ che ha a che fare esclusivamente col Marino e non va oltre. La divisione per capi, la ripetizione di certe "formule" d'occasione lo lega sì alle lettere secentesche, ma questa divisione è un segno del suo gioco sperimentale con vari registri e non l'intento di fuggire nell'anonimato.

La vita narrativizzata del Marino rimane il come, anzi - il come diventa il cosa - ed è per questo che felicemente si adatta a qualsiasi forma. Ciò hanno perfettamente capito i suoi biografi, rimanendo fedeli, pur con obiettivi

diversi ruoli sul mercato librario - come modelli linguistico-stilistici, modelli di vita, fonti di informazioni sui loro autori (e anche sugli editori, a volte figure chiave per il libro) ed altri ancora. Tutte le lettere conservano gli elementi integranti della forma, quali la data, il luogo, il nome del mittente e del destinatario. Col tempo però, già dagli ultimi decenni del Cinquecento, la situazione cambia. La lettera perde la propria funzione principale - quella comunicativa - e diventa una specie di raccolta di formule pronte per ogni occasione, senza data, senza indicazioni del luogo, persino senza firma, poiché in molti casi viene scritta a nome d'altri e la mano che si occupa della stesura sarà prevalentemente quella del Segretario. Alcuni studiosi vedono il punto di svolta nel 1590, l'anno della pubblicazione delle Lettere di Stefano Guazzo, poiché esse comportano un nuovo modus legendi, una nuova disposizione all'interno delle lettere: la classificazione in capi. Già dal frontespizio delle lettere di Guazzo si nota la loro diversa disposizione e quindi diversa funzione. Sono «Ordinate sotto i capi seguenti. Di raguagli. Di lode. Di raccomandazione. Di essortatione. Di ringratiamenti. Di congratulatione. Di scusa. Di consolatione. Di complimenti misti».

${ }^{33}$ A. QuONDAM, Le «carte messaggere»: retorica e modelli di comunicazione epistolare: per un indice dei libri di lettere del Cinquecento, Roma, Bulzoni, 1981, p. 32.

${ }^{34}$ Guglielminetti parla proprio della «carenza di commenti morali o politici agli avvenimenti del primo decennio del secolo XVII, carenza che colpisce subito il lettore di quest'epistolario») (GuglielminetTI, Tecnica e invenzione nell'opera di Giambattista Marino, cit., p. 20). 
magari diversi, ${ }^{35}$ a questo come mariniano. ${ }^{36}$ Se il modo del rappresentare diventa la sostanza dell'evento, allora i limiti realtà/storiografia/finzione sono definitivamente superati. Pertanto, $i$ "fatti" biografici ricavati, compreso questo, sarebbero forse meglio capiti se osservati nella chiave di lettura offerta dal Marino stesso: dal punto di vista esclusivamente letterario.

\author{
Nataša Gavrilović \\ TRANSPOZICIJA ISTORIJSKE STVARNOSTI U PISMIMA \\ ĐAMBATISTE MARINA
}

(Rezime)

Analizirajući jedno od najpoznatijih pisama Đambatiste Marina, i u kontekstu njegove epistolarne produkcije i epistole tout court, gde barokni pesnik opisuje atentat čijom je žrtvom bio, autorka rada ukazuje na potpuno preplitanje onoga što bi bila biografska, činjenično-istorijska dimenzija opisa događaja i samog posmatranja i interpretiranja te dimenzije u literarnom, retorizovanom ključu, te zaključuje da biografski podaci dobijaju svoje puno značenje tek ukoliko su interpretirani s književne tačke gledišta.

${ }^{35}$ Cfr. «Così, ritenendo d'interpretare le volontà dell'autore in punto di morte, ma in realtà cercando soprattutto di garantire se stessi e la loro personalità di poeti nuovi, i marinisti iniziavano subito dopo la morte del loro maestro quel processo di mistificazione della sua esistenza, che avrebbe fra poco trovato il maggior esito nelle prime raccolte delle sue lettere» (Ivi, p. 51).

${ }^{36}$ Lo dimostra anche un'osservazione dello Stigliani, nemico giurato del Marino, che in una sua lettera a F. Balducci, parlando della biografia mariniana del Baiacca, si lamenta che «ella non è una istoria, ma una favola ed una poesia in prosa» (MARINO, Epistolario (...), cit., p. 317). 